Mittheilung aus dem chemischen Laboratorium der Königl. Universität Erlangen.

\title{
Ueber das Atomgewicht des Tellurs $\mathrm{II}^{1}$ ); von A. Gutbier.
}

(Eingelanfen am 11. August 1905.)

Nach den von A. Ladenburg ${ }^{2}$ ), P. Köthner und E. A euer ${ }^{3}$ ) sowie von G. P. Baxter ${ }^{4}$ ) neuerdings ausgeführten Atomgewichtsbestimmungen des Jodes dürfte wohl kein Zweifel mehr darüber bestehen, dass das Atomgewicht des reinen Jodes erheblich niedriger ist, als dasjenige des nach den bisher bekannt gewordenen Untersuchungen ${ }^{5}$ ) für rein $z u$ haltenden Tellurs.

Angesichts dieser Thatsache kann man allerdings leicht dazu bewogen werden, entweder die Gültigkeit des periodischen Systems der Elemente in seiner bisher allgemein üblichen Fassung anzuzweifeln und das Tellur, wie H. Erdmann ${ }^{6}$ ) vorgeschlagen hat, unter die Platingruppe einzureihen, oder aber die Einheitlichkeit des Tellurs in Frage zu stellen, wie ja bereits von B. Brauner ${ }^{7}$ ) geschehen ist.

Um zur experimentellen Entscheidung über diese so ausserordentlich wichtige Frage mit beizutragen, habe ich eine Neubestimmung des Atomgewichtes des Tellur ausgeführt und zu diesem Zwecke Tellur vom Atomgewichte 127,6 einer bisher

1) Vergl. diese Amnalen $\mathbf{3 2 0}, 52$.

2) Ber. d. deutsch. chem. Ges. $\mathbf{3 5}, 2275$.

3) Diese Annalen 337, 1.

4) Proc. of the Americ. Acad. of Arts and Sciences $\mathbf{5 0}, 8$.

$\left.{ }^{5}\right)$ Ueber die Atomgewichtsbestimmungen des Tellurs sind in den letzten Jahren so viel Uebersichten publicirt worden, dass ich mir die Anfiihrung der einzelnen Arbeiten ersparen kann.

i) Lelirbuch d. anorg. Chemie Seite 721 und 726.

j) Monatsh. f. Chem. 10, 411. 
noch nicht in Anwendung gebrachten Reinigungsmethode unterworfen.

Ueber die Ausführung dieser bereits im Jahre 1903 abgeschlossenen Untersuchung und über die dabei erbaltenen Resultate in aller Kürze zu berichten ${ }^{8}$ ) ist der Zweck der folgenden Zeilen.

\section{Ceber die zur Reinigung des Tellurmaterials angewandten Methoden.}

(Experimentell mitbearbeitet von $W$. Wagenknecht. ${ }^{9}$ )

Aus dem Ausgangsmaterial — zunächst benutzte ich ungarisches Rohtellur, später 95 procentiges Tellur von Dr. The odor Schuchardt in Görlitz - wurde das Tellur in der schon früher beschriebenen Weise ${ }^{10}$ ) durch Reduction mit Schwefeldioxyd abgeschieden und nach dem Auswaschen bei niederer Temperatur getrocknet.

Dann wurde das so gewonnene Tellur der Destillation im Vacuum unterworfen, indern das fein zerriebene trockne Material in Mengen von $5-7 \mathrm{~g}$ in enge, einseitig zugeschmolzene Verbrennungsröhren eingeschüttet und mit einem abgeplatteten Glasstabe fest zusammengedrückt wurde; nachdem die Röhren mit je zwei Pfropfen von besonders gereinigtem Asbest ${ }^{12}$ ) so

s) Eine ausführliche Schilderung der vorliegenden Untersuchung erscheint demnächst in den sitzungsberichten der physikalischmedicinischen Societät in Erlangen.

9) Die Inaugural-Dissertation von W. Wagenknecht enthält eine erschöpfende Beschreibung der einzelnen Versuche.

to) Vergl. z. B. A. Gutbier, Studien über das Tellur (Leipzig 1902), Seite 11 .

11) Vergl. G. Pellini, Ber. d. deutsch. chem. Ges. 34, 3807; P. Köthner, diese Annalen :319, 1; sowie Georg W. A. Kahlbaum, R. Rotlı und P. Siedler, Zeitschr. f. anorg. Chem. $\mathbf{2 9}, 177$.

12) Betr. der Angaben über die Reinigung des Asbestes und die Reindarstellung der Reagentien verweise ich auf die Dissertation von 
beschickt worden waren, dass der eine Pfropfen fest auf dem Tellur lag, während der zweito in angemessener Entfernung angebracht war, wurden sie mit Hülfe einer Quecksilberluftpumpe, der ein mit echtem Blattgold beschicktes Kugelrohr vorgelegt war, auf $2-3 \mathrm{~mm}$ evacuirt und schliesslich in der von $\mathrm{P}$. Köthner ${ }^{13}$ ) beschriebenen Weise so lange erhitzt, bis das Tellur durch die beiden Asbestpfropfen hindurchdestillirt war.

Zur Ueberführung. des destillirten Tellurs in eine salzartige, durch Umkrystallisiren leicht $\mathrm{zu}$ reinigende Verbindung ${ }^{14}$ ) schlug ich aus besonderen Gründen zwei Wege ein, indem ich einen Theil des Materials in die Tellursäure, den anderen in das basische Tellurnitrat verwandelte.

Die Tellursäure wurde, da zu jener Zeit das Hydroperoxydverfahren ${ }^{15}$ ) noch nicht ausgearbeitet war, nach der Methode von L. Staudenmaier ${ }^{16}$ ) unter Beobachtung aller von mir bereits gegebenen Vorsichtsmassregeln ${ }^{17}$ ) und unter einer geringfügigen Modification des Darstellungsganges folgendermassen bereitet:

Um die von F. Flury und mir ${ }^{18}$ ) beobachtete Abscheidung von überschüssigem Chrominitrat zu vermeiden, wurde die Lösung des fein gepulverten Tellurs in der reinsten Salpetersäure

W. Wagenknecht und auf meine demnächst erscheinende Abhandlung.

13) loc. cit.

14) Bekanntlich hat P. Köthner, loc. eit., spectralanalytisch nachgewiesen, dass das Tellur auch noch nach der Vacuumdestillation Verumreinigungen enthält, welche nur dadurch entfernt werden können, dass man das Material in salzartige Verbindungen überfiihrt.

t5) Vergl. A. Gutbier und W. Wagenknecht, Zeitschr. f. anorg. Chem. 40, 260 und A. Gutbier und F. Resenscheck, ebenda $4 \mathbf{2}, 174$.

16) Zeitschr. f. anorg. Chem. 10, 189.

1i) Vergl. besonders Zeitschr. f. anorg. Chem. B2, 96.

18) Zeitschr, f. anorg. Chem. \$2, 96. 
auf dem Wasserbade zur Trockne verdampft, der Rückstand sorgfältig bei $105-110^{\circ}$ getrocknet und dann fein zerrieben in der concentrirten reinsten Salpetersäure suspendirt.

Als nun das Gemisch mit einer klaren verdünnten Lösung von Chromsäure in der Siedehitze behandelt wurde, war es thatsächlich möglich, jeglicheu Ueberschuss an Chromsäure zu vermeiden. Die Oxydation konnte nämlich als beendet angesehen werden, wenn die letzte Spur des Tellurdioxydes in Lösung gegangen war. Die braune Farbe der Lösung änderte sich dann selbst bei halbstündigem Erhitzen nicht mehr und bei dem Einengen der Lösungen schied sich die Tellursäure so gut ab wie nie zuror.

Die Tellursäure wurde nun in bekannter Weise ${ }^{19}$ ) weiter behandelt und nach dem auf dem Wasserbade erfolgten Erhitzen der Krystalle mit heissem Wasser soweit als möglich in Lösung gebracht; die so gewonnenen Flüssigkeiten wurden mehrmals durch gehärtete Filter filtrirt, um die geringen Mengen der beim Trocknen der Krystallmasse immer entstehenden wasserärmeren Hydrate der Tellursäure ${ }^{20}$ ) sicher zu entfernen.

19) Vergl. I. Standenmaier, loc. cit. und A. Gutbier, loc. cit.

20) Derartige in Wasser nicht leicht auflösbare Riickstände treten, wie ich mit F. Flury (Zeitschr. ft. anorg. Chem. 32, 96) zeigen komute, bei nicht vollkommen reiner Tellursäure leicht auf und riihren von den von F. Mylius (Ber. d. deutsch. chem. Ges. 34, 2208) zuerst beschriebenen, "wasserïrmeren Hydraten der Tellursäure" her. Diese Producte entstehen, wie wir erkannt haben, besonders leicht bei dem Einengen wässriger Lösungen von unreiner Tellursäure auf dem Wasserbade und werden stets erhalten, wenn eine wässrige Lösung von selbst reinster T'ellursäure bei höherer Temperatur zar Trockne verdampft wird und die Masse glasartig erstarrt; ausserdem werden sie bei dem Trocknen feuchter. Tellursäurekrystalle auf dem Wasserbade gebildet.

Die wasscrärmeren Hydrate treten, wie wir mitgetheilt haben, in zwei Modificationen auf, von denen die eine so fcin ist, dass sie selbst bei tagelangem Stehen der Flüssigkeit nicht zum $\mathrm{Ab}$ sitzen zu bringen ist, während die andere Form, die übrigens auch allem Auscheine nach I'. Köt h ner (loc. cit.) in seiner Tellursäture 
Die klaren Lösungen wurden in Platinschalen der freiwilligen Krystallisation überlassen und die ausgeschiedenen Krystalle noch zehnmal aus wenig Wasser unter Störung der Krystallisation umkrystallisirt.

Die schliesslich erhaltenen klaren und farblosen Krystalle gingen beim Uebergiessen mit reinem heissen Wasser in Lösung, ohne die geringste Spur eines Rückstandes zu hinterlassen.

Schliesslich warde die im Vacuumexsiccator getrocknete Tellursäure in der schon früher beschriebenen Weise ${ }^{21}$ ) in Tellurdioxyd übergeführt, das von der reinen warmen Salzsäure ohne die geringste Chlorentwickelung aufgelöst wurde und auch beim Kochen der Lösungen kein Chlor lieferte. Das Tellurdioxyd enthielt also kein Trioxyd.

Der andere Theil des destillirten Tellurs wurde unter peinlichst genauer Einhaltung aller der von P. Köthner ${ }^{22}$ ) gegebenen Vorsichtsmassregeln in das basische Tellurnitrat übergeführt, welches durch zweimaliges Umkrystallisiren gereinigt, mit absolutem Alkohol behandelt und nach dem Trocknen in gleicher Weise in Tellurdioxyd verwandelt wurde.

Auch das hier erhaltene Präparat entwickelte mit Salzsäure kein Chlor; es war also ebenfalls frei von Tellurtrioxyd und Salpetersäure.

Die gereinigten Präparate wurden in Salzsäure gelöst und die mit Wasser verdünnten Lösungen nach dem Filtriren in der Siedehitze mit reiustem arsenfreien Schwefelwasserstoff gesättigt.

Der Grund für dieses Vorgehen ist in folgenden Ueberlegungen zu suchen: Bekanntlich ${ }^{23}$ ) ist der aus Tellurlösungen

gehabt hat, eine voluminöse Gallerte bildet, welche sich relativ leicht durch Filtriren entfernen lässt.

a1) Diese Annalen $\mathbf{3 2 0}, 56$.

20) 100 cit.

${ }^{23}$ ) Vergl. Berzelius, Jelub. f. Chemie; Becker, diese Amnalen 180, 260; B. Bra uner, Journ. chem. Soc. 67, 5́4ó; L. Stauden- 
gefällte Niederschlag sehr unbeständig; mit F. Flury habe ich gezeigt ${ }^{24}$ ), dass einerseits dem aus Tellurdioxyd entstehenden Producte die Formel $\mathrm{TeS}_{2}$ zukommt, dass es aber andererseits nur als ein Gemenge in dem constanten Verhältnisse $1 \mathrm{Te}: 2 \mathrm{~S}$ aufzufassen ist, da wir den Schwefel durch Extraction mit Schwefelkohlenstoff bis zu 1,18 pC. entfernen konnten ${ }^{25}$ ).

Da weitere Versuche zeigten, dass hiermit die Grenze des extrahirbaren Schwefels erreicht war, erwähnten wir bereits zweierlei Deutung dieser interessanten Thatsache: es könnte nämlich entweder ein Gleichgewichtszustand eingetreten, oder aber dieser zurückgehaltene Schwefel, wie ja schon B. Brauner ${ }^{26}$ ) vermuthete, chemisch an ein anderes Element gebunden sein, das eine grosse Affinität zum Tellur besitze.

Zur Entscheidung dieser hochwichtigen Frage stellte ich mir 'Tellurschwefel dar, um dieses Product weiter geeigneten Prozessen unterwerfen zu können.

Die Niederschlage wurden mit frisch bereitetem Schwefelwasserstoffwasser und dann mit lauwarmem Wasser bis zur neu-

maier, loc. cit.; H. Rose, Poggendorff's Ammalen 112, 316; A. Gutbier, "Studien über das Tellur", Seite 40; A. Gutbier, und F. Flury, Zeitschr. f. anorg. Chem. 32, 272.

24) 10c. cit.

${ }^{25}$ ) Ergänzend ist zu bemerken, dass der Schwefelgehalt des Rückstandes nach 36 stiindiger Extraction im Soxleth-Apparate manchmal noch iiber 2 pC. beträgt, da der Niederschlag sieh leicht zusammenballt und somit schwer gleichmässig extrahiren lässt; wird aber das Product nochmals fein zerrieben 6-12 Stunden extrahirt, so enthält der Rückstand num nur noch 1,18 bis $1,2 \mathrm{pC}$. S. Folgende Analysen mögen das beweisen:

1) Rückstand nach 36 stïndiger Extraction:

a. $0,2580 \mathrm{~g}$ gaben $0,0433 \mathrm{BaSO}_{4}=2,30 \mathrm{pC}$. $\mathrm{s}$.

b. $0,4030 \mathrm{~g} \quad, \quad 0,0694 \mathrm{BaSO}_{4}=2,36 \mathrm{pC}$. s.

2) Rückstand nach erneuter 12stiindiger Extraction:

a. $0,6297 \mathrm{~g}$ gaben $0,0551 \mathrm{BaSO}_{4}=1,20 \mathrm{pC}$. S.

b. $1,0300 \mathrm{~g} \quad, \quad 0,0900 \mathrm{BaSO}_{4}=1,19 \mathrm{pC}$. $\mathrm{s}$.

26) loc. cit. 
tralen Reaction decantirt, durch Aufsaugen auf ein Filter nach Möglichkeit von der anhängenden Flüssigkeit befreit und bei $105^{\circ}$ getrocknet, hierauf so fein als möglich pulverisirt und in Portionen von $10-15 \mathrm{~g}$ im Soxleth'schen Extractionsapparate mit einem dreifach fractionirten und dann rectificirten Schwefelkohlenstoff 36 Stunden lang behandelt. Der Rückstand wurde nach dem Abwaschen ${ }^{27}$ ) und 'Trocknen fein gepulvert abermals 12 Stunden lang extrahirt und nach dem Abwaschen bei $70^{\circ}$ getrocknet.

Es galt nun, aus djesem Materiale das reine Tellur zu isoliren und so versuchte ich schliesslich nach einer grossen Reihe von Misserfolgen, den Extractionsrücksand der Destillation im Vacuum zu unterwerfen. Diese wurde mit je 5 --10 $\mathrm{g}$ in der oben beschriebenen Weise ausgeführt.

In der That gelang es mir, durch 14 malige fractionirte Destillation der einzelnen Portionen im Vacuum reines, absolut schwefelfreies Tellur zu erhalten.

Die hierbei gesammelten Beobachtungen ${ }^{28}$ ) lassen sich kurz folgendermassen zusammenfassen: Bei den ersten vier Destillationen war die Menge des Rückstandes und des verflüchtigten Schwefels relativ gross, während andererseits in dem Destillate Schwefel noch mit Sicherheit nachgewiesen werden konnte. Von der fünften Destillation ab - das Tellur wurde jetzt durch vier Asbestpfropfen destillirt - konnte weder ein Rückstand noch Schwefel in der Röhre oder im Destillate nachgewiesen werden; aber immer war in deutlich sichtbaren Spuren ein weiss gefärbter Beschlag in den Röhren zu bemerken, der bis zur zwölften Destillation immer wieder auftrat, ohne dass es uns trotz wiederholter, mühevoller Versuche gelungen wäre, seine chemische Natur zu erklären, da der Beschlag meist nur einem Hauche glich.

27) Vergl. A. Gutbier und F. Flury, loc. eit.

28) Ausführliche Schilderung siehe in der Dissertation von W. Wa genknecht. 
Wir haben schliesslich das Material noch zweimal destillirt und constatiren können, dass weder der geringste Rückstand, noch auch die minimalsten Spuren eines Beschlages auftraten.

Dieses Tellurpräparat bestand aus schmalen, leicht zerbrechlichen Metallstücken, die einen herrlichen Metallglanz besassen und an den Bruchstellen die gleiche ausgezeichnete Krystallstructur besassen, die oft an der Oberfläche der Reguli zu bemerken war; selbst mit den feinsten analytischen Methoden konnte nicht die geringste Spur von Schwefel oder anderen Verunreinigungen in dem Präparate nachgewiesen werden.

\section{Die Bereitung des Analysenmaterials.}

Das Tellur musste nun in eine durch Umkrystallisiren leicht zu reinigende Verbindung übergeführt werden, mit welcher die Atomgewichtsbestimmung vorgenommen werden konnte.

Nach mehreren orientirenden Versuchen entschied ich mich schliesslich für die Darstellung des basischen Tellurnitrats und zwar nicht allein aus dem Grunde, weil P. Köthner ${ }^{29}$ ) mit diesem Producte so vorzüglich übereinstimmende Resultate erhalten hatte, sondern vor allen Dingen deshalb, weil ich zur Bereitung dieser Verbindung ausser der reinsten Salpetersäure und dem zu ibrer Verdünnung benöthigten reinstem Wasser keinerlei andere Reagentien benöthigte, durch welche mein Tellurpräparat hätte wieder verunreinigt werden können.

Zur Darstellung des basischen Tellurnitrats habe ich den ron $\mathrm{P}$. Köthner ${ }^{30}$ ) ausführlich beschriebenen Weg eingeschlagen; ich kann mich deshalb, um so mehr als eine ausführliche Beschreibung dieses Verfahrens in meiner ausführlicheren Mittheilung gegeben wird, auf folgende kurze Angaben bescbränken.

Um ganz sicher zu vermeiden, dass Staub u. s. w. in die Flüssigkeiten gelangen könnte, wurden die Krystallisationsschalen mit V. Meyer'schen Glastrichtern bedeckt; das Einengen der

29) loc. cit.

30) loc. cit. 
Lösungen fand in einem besonderen Raume statt, so dass die Einwirkung schädlicher Dämpfe unmöglich war.

Die erhaltenen Krystalle wurden nach dem Abspülen zweimal umkrystallisirt und dann in der von $P$. Köthner vorgeschriebenen Weise gereinigt, ohne dass je Abscheidung von Tellurdioxyd oder von telluriger Säure bemerkt worden wäre. Das mit absolutem Alkohol behandelte Product wurde in einer kohlensäurefreien Atmosphäre abgesaugt und schliesslich im evacuirten Exsiccator bis zur Gewichtsconstanz getrocknet.

Im trocknen Zustande zeigte das Präparat vollkommenen Glanz und erwies sich bei der Betrachtung unter dem Mikroskope als durchaus einheitlich und klar; auch bei dem Erhitzen auf $120^{\circ}$ nahm keine Probe an Gewicht ab; das Präparat war also frei von Tellurdioxyd.

Da ich aber trotz aller erdenklichen Mühe und Sorgfalt mit dem basischen Tellurnitrate übereinstimmende Resultate nicht habe erhalten können ${ }^{31}$ ), stellte ich mir folgendermassen Tellurdioxyd dar:

Eine angemessene Menge des Nitrates wurde in einem mit gat schliessendem Deckel versehenen Platintiegel, der in einem Berliner Porzellantiegel stand und mit diesem in den runden Ausschnitt einer Asbestplatte gehängt war, in der ron P. Köthner bescbriebenen Weise durch Erhitzen mit einer kleinen Flamme zersetzt; die Temperatur wurde ganz langsam erhöht und der Tiegel in bestimmten Zeitabschnitten nach einstündigem Abkühlen gewogen. Das Erhitzen wurde erst dann beendet, wenn drei aufeinander folgende Wägungen Gewichtsconstanz anzeigten.

Das so gewomnene Dioxyd bildete eine rein weisse, fast porös zu nennende Masse, welche, wie ich es beabsichtigt hatte, nicht geschmolzen war und sich leicht zu einem feinen Pulver zerreiben liess $^{32}$ ).

31) Vergl. meine diesbeziiglichen Mittheilungen a. a. 0.

32) Ich wendete nicht, wie z. B. G. Pellini (loc. cit.) geschmolzenes Tellurdioxyd an, weil das schmelzende Dioxyd, wie P. Köthner 
Durch die rein weisse Farbe war erwiesen, dass das Präparat weder mit Platin noch mit Tellur verunreinigt war, denn dadurch würde eine schwach grane bezw. bräunliche Färbung hervorgerufen worden sein; ausserdem lösten sich $5,00378 \mathrm{~g}$ in mässig verdünnter Salzsäure ohne Rückstand auf, was durch die Gewichtscontrolle eines $\mathrm{N}$ e u bauer-Tiegels, durch den ich die Lösung filtrirte, festgestellt wurde. Durch Lösen von $3,1270 \mathrm{~g}$ des Dioxydes in Salzsäure wies ich auch nach, dass das Präparat nicht mit Trioxyd oder Salpetersäure verunreinigt war, denn Beimengungen dieser Art hätten bei dem Auflösen Chlor entwickeln müssen.

Da schliesslich auch noch genau abgewogene Proben des Dioxydes bei dem Schmelzen in doppelten Platintiegeln keine Gewichtsveränderung erlitten, konnte ich davon überzengt sein, dass ich chemisch reines Tellurdioxyd zur Verfügung hatte.

\section{Ueber die Inalysenmethoden und die erhaltencn Resultate.}

Die Methoden der Atomgewichtsbestimmung waren durch die Eigenschaften des Analysenmateriales gegeben und bestanden in der Bestimmung des Tellurgehaltes.

Die Wägungen wurden auf der bereits beschriebenen ${ }^{33}$ ) Wage mit einem corrigirten Gewichtssatze ausgeführt; die Analysenangaben sind auf den luftleeren Raum reducirt.

\section{A. Reduction des Tellurdioxydes mittelst Wässerstoff.}

Die Reduction wurde nach L. Staudenmaier ${ }^{34}$ ) bei Gegenwart von feinst vertheiltem, nach Stas bereitetem Silber und von reinstem, aus Bergkrystall gewonnenem Quarzsand ausgeführt $\left.^{35}\right)$.

angiebt, Spuren von Platin ans dem Tiegel anfiöst, und weil es sich nur schwierig zu einem feinen Pulver zerreiben lässt.

39) Diese Annalen 320, 52.

${ }^{34)}$ Zeitschr. f. anorg. Chem. 10, 189.

35) Sowohl das Silber als auch der Quarzsand erlitten bei dem im Wasserstoff and dam im Vacuum erfolgten Erhitzen keine Gewichtsverïnderung; beide Präparate waren also rein. 
Das Tellurdioxyd wurde in ein Porzellanschiffehen eingewogen und mit Hülfe eines Platindrahtes, der dann in der Mischung verblieb, innig mit der acht- bis zehnfachen Menge des zur Bildung von Silbertellurid erforderlichen Silbers und mit einer genügenden Menge Quarzsand vermischt; schliesslich wurde die Mischung noch mit Silber bedeckt. Das Schiffchen wurde mit einem zweiten, nur mit Quarzsand und Silber beschickten, ebenso genau gewogenen Schiffchen und einer Silberspirale in das durch ein Glasschiff mit dem Gasentwickelungsund Reinigungsapparate verbundene Reductionsrohr eingeführt.

Der Wasserstoff wurde aus reinstem, arsenfreiem, mit Platinschnitzeln gemengtem Zink mit chemisch reiner Schwefelsäure bereitet und nach einander durch concentrirte Kalilauge, Silbernitratlösung, Kaliumpermanganatlösung und chemisch reine concentrirte Schwefelsäure geleitet; er trat dann durch eine mit platinirtem Asbest gefüllte Kugelröhre in ein langes, mit Kupferspiralen angefülltes, rothglühendes Rohr und wurde durch concentrirte, chemisch reine Schwefelsäure und zuletzt durch Phosphorpentoxyd getrocknet.

Die Reduction wurde zunächst im Luftbade bei $100^{\circ}$ eingeleitet; dann wurde das Rohr auf $230^{\circ}$ und über $270^{\circ}$ auf $350^{\circ}$ erhitzt, unter Wasserstoff erkalten gelassen und schliesslich auf einem Verbrennungsofen nach und nach bis zur dunklen Rothgluth erbitzt. Dann liess man das Rohr wieder langsam erkalten, während ein stärkerer Wasserstoffstrom durch den Apparat geleitet wurde und wog die Schiffchen. Der ganze Prozess wurde so oft wiederholt, bis das mit dem Tellurdioxyd beschickte Schiffchen Gewichtsconstanz angenommen hatte; dies wurde meist schon bei der dritten Wiederholung erreicht und das Schiffchen änderte dann sein Gewicht auch nicht mehr, selbst wenn die Temperatur weiter gesteigert wurde.

Sobald Gewichtsconstanz erreicht war, wurden beide Schiffchen in dem evacuirten Reductionsrobre erhitzt; auch dieser Prozess wurde so lange wiederholt, bis das Gewicht constant blieb. 
Das zweite, nur mit der Silber-Quarzsand-Mischung beschickte Schiffchen hatte sein Gewicht nicht verändert und ebenso blieb die vorgelegte, natürlich weniger hoch erhitzte Silberspirale blank: ich erblicke in dieseu Thatsachen ein erfreuliches Zeichen dafür, dass einerseits mein Silber und Quarzsand den erforderlichen Reinheitsgrad besassen und dass andererseits nicht die geringste Spur von Tellur bei der Reduction verflüchtigt wurde.

Was die Methode selbst anbetrifft, so zeigen die Resultate, dass sie sehr zuverlässig ist; der einzige, ron mir empfundene Mangel besteht darin, dass man durch die Grössenverhältnisse der gebräuchlichen Porzellanschiffchen an ziemlich constante Substanzmengen gebunden ist. Ich habe mir daher sehr grosse Schiffchen anfertigen lassen und mit Erfolg die Substanzmengen in gewissen Grenzen variiren können.

Ich erhielt folgende Resultate:

\begin{tabular}{c|c|c|c|c}
\hline \hline Nr. & $\begin{array}{c}\text { Angewandt } \\
\text { Te0. }\end{array}$ & $\begin{array}{c}\text { Gefunden } \\
\text { Te }\end{array}$ & pC. Te & Atomgewicht \\
\hline 1 & $2,99688 \mathrm{~g}$ & $2,39585 \mathrm{~g}$ & 79,944 & 127,55 \\
2 & $1,30740 \mathrm{~g}$ & $1,04527 \mathrm{~g}$ & 79,950 & 127,60 \\
3 & $2,04325 \mathrm{~g}$ & $1,63380 \mathrm{~g}$ & 79,955 & 127,68 \\
4 & $2,61725 \mathrm{~g}$ & $2,09249 \mathrm{~g}$ & 79,949 & 127,59 \\
5 & $3,61725 \mathrm{~g}$ & $2,89222 \mathrm{~g}$ & 79,956 & 127,65
\end{tabular}

B. Reduction des Tellurdioxydes mittelst Hydrazin.

Diese Methode, welche mir schon zur Ausführang meiner ersten Atomgewichtsbestimmungen gedient hatte ${ }^{36}$ ), habe ich auch jetzt wieder angewandt, nachdem ich mich während meiner Untersuchungen über das Tellur unausgesetzt von ibrer Exactheit und Brauchbarkeit habe überzeugen können, und nachdem es mir dank der treuen Unterstützung seitens einiger Mitarbeiter gelungen ist, alle etwa auftretenden Fehlerquellen zu studiren und zu umgehen!

3e) Diese Aunalen 320, 52 . 
Meine diese Methode betreffenden Erfahrungen habe ich bereits ausführlich publicirt ${ }^{37}$ ); ich kann daher auf diese $\mathrm{Ab}$ handlung, in der ich mich besonders über die Oxydationsfähigkeit des gefällten Tellurs ausgesprochen habe, verweisen und zur Beschreibung des diesmal angewandten Analysenganges übergehen.

Das Tellurdioxyd wurde in eine Platinschale ${ }^{38}$ ) eingewogen, welche mit dem festschliessenden Deckel verschlossen und auf ein nicht angeheiztes Wasserbad ${ }^{39}$ ) gestellt wurde; dann wurde die Substanz bei gewöhnlicher Temperatur in der nöthigen Menge mässig verdünnter reinster Salzsäure gelöst.

Zur Reduction wurde das Zehnfache der theoretisch erforderlichen Menge von frisch bereitetem Hydrazinchlorhydrat $^{40}$ ) in Wasser gelöst und der Tellurlösung in Por-

32) Sitzungsberichte der physikalisch-medicinischen Societät zu Erlangen $\mathbf{3 t i}, \mathbf{1 3 0 .}$

3s) Diese war vorher ausgegliht und in einem zu dieseu Zwecken besonders construirten Trockenschiranke unter Wasserstoff bei $105^{\circ}$ bis zu constantem Gewicht erhitzt.

39) Die Porzellanringe, auf welche die Schale gestellt wurde, waren mit den Streifen eines seidenen Tuches in vierfacher Lage umhüllt.

40) Ich hatte frïher bei Verwendung des Hydrazinhydrates die unangenehme Erfahrung gemacht, dass eine geringe Menge Tellur an dem Boden der Schale, wie auch an dem Deckel so fest anhaftete, dass sic durch Abspritzen mit Wasser nicht zu entfernen war und nur unter gan $z$ besonderen Bedingungen zur Wägung gebracht werden konnte. Obwohl nene Controllversuche bewiesen haben, dass so wirklich einwandfreie Resultate erhalten werden, habe ich mich dennoch bemüht, alle etwa auftretenden Fehlerquellen mit Sicherheit auszuschliessen und deshalb das weniger energisch aber ebenso quantitativ reducirend wirkende Hydrazinchlorhydrat verwandt.

Aber auch jetzt haftete immer noch eine geringe Menge Tellur sehr fest an der Schale, weun das Reductionsgemisch auf einem Asbestteller direct erhitzt wurde. Auch diesen Uebelstand habc ich durch einen kleinen Kunstgriff beseitigen können. Wenn man nämlich das Reductionsmittel zuerst bei gewöhnlicher Temperatur einwirken lässt, dann den Schaleninhalt nach und nach auf dem 
tionen ron je $5 \mathrm{ccm}$ am Ausguss der bedeckten Schale so zugefügt, dass ein weiterer Zusatz von $5 \mathrm{ccm}$ jedesmal erst nach 25 Minuten erfolgte. Dann wurde das Wasserbad allmählich angeheizt, die Temperatur eine Stunde bei $50^{\circ}$ gehalten und hierauf weiter gesteigert, bis das Wasser schliesslich eine Stunde im Sieden erhalten wurde.

Unter diesen Bedingungen war stets vollständige Reduction eingetreten. Die bedeckte Schale wurde daun auf einer Asbestplatte nach und nach bis zum Sieden der Flüssigkeit erhitzt; nach dem Erkalten gab ich nocbmals $5 \mathrm{ccm}$ der Hydrazinchlorhydratlösung hinzu und sah nach 25 Minuten nach, ob eine Färbung der Flüssigkeit eingetreten war. Dies war bei keiner Analyse der Fall, denn ich hatte niemals verabsäumt, den Rand der Schale und den Deckel öfters mit geringen Mengen Wasser abzuspülen.

Nach nochmaligem Aufkochen liess ich so weit erkalten, bis ich die Schale bequem anfassen konnte, spülte dann den Deckel, an dem sich nur bei der zweiten Analyse ganz minimale, mit Wasser leicht entferubare Spuren von Tellur befanden, ab und benutzte zum Filtriren einen Neubauer-Platintiegel, der in dem durch die umstehende Figur veranschanlichten Apparate ${ }^{41}$ ) in einem besonders construirten Trockenschranke

Wasserbade erwärmt und schliesslich über freier Flamme bis zum Sieden erhitzt, so setzt sich - allerdings nur, wenn die Temperatur sohr vorsichtig gesteigert wird - nicht die geringste spur von Tollur an dem Boden der Schale fest.

$\left.{ }^{41}\right)$ Zur Erklärung der Figur sei mitgetheilt, dass der Apparat aus cinem grossen, aus schwer schmelzbarem Glase gefertigten Wägeglase $A$ besteht, das ich in der Mitte durchschnitt und unten trichterförmig auszog; in die Oeffnung $B$ blies ich einen kleinen Trichter $C$ an, auf dessen umgebogenen Rand eine Porzellansiebplatte unbeweglich liegt. Da durch den Apparat Wasserstoff geleitet werden musste, wurde einerseits in den Deckel bei $E$ das zur Ableitung dienende Glasrohr $F$ eingeschmolzen, während das Ende des ausgezogenen Glases ebenfalls nmgebogen wurde und als Gaszuleitnngsrohr $D$ dient. Durch einen gläsernen Dreifuss, 
zuerst im Wasserstoffstrome und dann im Vacuum bei $105^{0}$ bis zur Gewichtsconstanz getrocknet worden war.

Das Tellur wurde zuerst mit lauwarmem Wasser bis zur neutralen Reaction decantirt, dann auf den Tiegel übergeführt und weiter ausgewaschen; unter Einhaltung aller Bedingungen

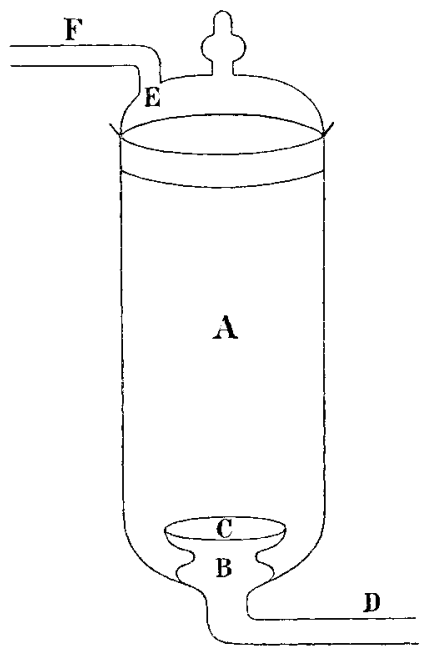

Fig. I.

wurde jeder Niederschlag vollständig aus der Schale in den Tiegel gebracht.

Nach dem Festsaugen des Niederschlages wurde der Tiegel unter den gleichen Bedingungen wie vor der Benützung in dem Apparate bis zu constantem Gewichte getrocknet.

Das gesammte Filtrat wurde in der Platinschale mit $30 \mathrm{ccm}$ einer 20 procentigen Hydrazinhydratlösung versetzt und zunächst auf dem Wasserbade und dann über kleiner Flamme auf $25 \mathrm{ccm}$

der an einer Seite eine kreisrunde, zur Aufnahme von $D$ dienende Oeffinung besitzt, wird der ganze Apparat festgehalten.

Dieser Trockenapparat wird jetzt ron Wagner \& $M$ un in München sowohl in Glas, als auch in jedem gewïnschten Metall geliefert. 
eingeengt; es war mir eine grosse Genugthuung, constatiren zu können, dass der Schaleninhalt sich hierbei nicht im Geringsten färbte oder dass gar eine Abscheidung erfolgte. Die Reduction war also vollständig gelungen!

Schliesslich habe ich auch noch die beiden Proben angestellt, welche mir beweisen mussten, ob ich auf mein Verfahren unbedingtes Vertrauen setzen könnte, oder nicht. Ich habe einerseits die nach dem Abfiltriren des eingedampften Filtrats sauber ausgewaschene Platinschale sammt Deckel unter den gleichen Bedingungen, wie vor der Reduction erhitzt, ohne Gewichtszunahme constatiren können; andererseits habe ich noch jedes Mal nach der letzten Wägung den Tiegelinhalt mit warmer Salzsäure behandelt, diese abgesaugt und näher untersucht. Die Säure blieb ungefärbt und lieferte auch bei dem Erhitzen mit überschüssigem Hydrazinhydrat keine Färbung.

Auf Grund aller dieser von mir erhaltenen Resultate darf ich annehmen, dass ich bei der von mir angewandten Sorgfalt vollkommen einwandfreie Resultate erhalten habe.

Die Bestimmungen lieferten folgende Zahlen:

\begin{tabular}{c|c|c|c|c}
\hline \hline Ne. & $\begin{array}{c}\text { Angewandt } \\
\text { TeO }\end{array}$ & $\begin{array}{c}\text { Gefunden } \\
\text { Te }\end{array}$ & pC. Te & Atomgewicht \\
\hline 6 & $1,90601 \mathrm{~g}$ & $1,52390 \mathrm{~g}$ & 79,952 & 127,62 \\
7 & $1,03532 \mathrm{~g}$ & $0,82784 \mathrm{~g}$ & 79,959 & 127,67 \\
8 & $2,2200 \mathrm{~g}$ & $1,77480 \mathrm{~g}$ & $79,94 \tilde{\mathrm{g}}$ & 127,05
\end{tabular}

\section{Discussion der Resultate.}

Im Laufe der vorliegenden Untersuchung habe ich also folgende Resultate erhalten:

$\Lambda$. bei der Reduction des Tellurdioxydes mittelst Wasserstoff:

$T_{e}=127,55 ; 127,60 ; 127,68 ; 127,59 ; 127,65$.

B. bei der Reduction des Tellurdioxydes mittelst Hydrazin:

$\mathrm{Te}=127,62 ; 127,67 ; 127,55$.

Im Nittel aus sämmtlichen acht Bestimmungen ergiebt sich für das Atomgewicht die Zahl: 127,61 . 
Diese Resultate zeigen, dass das Tellur auch durch einen solchen energischen Reinigungsprozess, wie ich ihn angewandt habe, nicht verändert worden ist. Dem Element ist also auch fernerhin noch das Atomgewicht 127,6 zuzuerkennen!

Was die von mir angewandten Methoden anbetriff, so kann ich mich sehr kurz fassen.

Dass die von $L$. Staudenmaie $\mathbf{r}^{42}$ ) ausgearbeitete Bestimmungsart, wenn sie vorschriftsmässig angewandt wird, vortreffliche Resultate zeitigt, hat uns die Arbeit von G. Pellini ${ }^{43}$ ) zuerst gezeigt und auch P. Köthner ${ }^{44}$ ) hat sich dahin geäussert, dass es ihm nicht nothwendig erschienen ist, die Bestimmungen Staudenmaier's in Anbetracht der sicheren Arbeitsmethode nachzuprüfen; ich bin auf Grund meiner eigenen Versuche davon überzeugt, dass diese Bestimmungsmethode einen sehr grossen Grad von Genauigkeit verbürgt.

So erblicke ich denn auch in den Resultaten meiner mittelst Hydrazinchlorhydrat ausgeführten Bestimmungen eine äusserst werthvolle Bestätigung der von mir nach dem Staudenmaierschen Verfahren erhaltenen Zahl, um so mehr, als ich redlich bemüht gewesen bin, mein Verfahren in einwandsfreier Weise auszugestalten.

\footnotetext{
42) Zeitschr. f. anorg. Chem. 10, 189.

43) loc. cit.

4) loc. cit.
} 